\title{
Dimensionality in Field Theory and in Spin Wave Theory
}

\author{
U. KÖBLER ${ }^{a, *}$ AND A. HOSER ${ }^{b}$ \\ ${ }^{a}$ Forschungszentrum Jülich, 52425 Jülich, Germany \\ ${ }^{b}$ Helmholtz-Zentrum Berlin, Hahn-Meitner Platz 1, 14109 Berlin, Germany
}

\begin{abstract}
The different meaning of dimensionality and universality in field theory and in spin wave theory is illustrated on account of experimental examples. In spin wave theory it is distinguished between the dimensionality of the spin and the dimensionality of the exchange interactions. According to Renormalization Group (RG) theory, these atomistic characteristics are unimportant for the critical dynamics. Instead by inter-atomic interactions the dynamics of the ordered state is determined by the excitations of the continuous magnetic medium. These excitations are bosons. Consequently, the dimensionality of ordered magnets has to be assessed to the dimensionality of the relevant boson field. The most serious consequence of RG theory is that the magnetic ordering transition also is executed by the boson field. Typical for boson dynamics is a finite width of the critical range. In the atomistic models universality applies asymptotically at $T_{c}$ only. It is evident that the critical power functions of the field dynamics are different from those of the atomistic dynamics.
\end{abstract}

DOI: 10.12693/APhysPolA.127.356

PACS: 75.10.-b, 75.30.Ds

\section{Introduction}

Since development of Renormalization Group (RG) theory we know that the critical dynamics in the vicinity of the magnetic ordering transition is as for a continuous medium. Surprisingly, spins and interactions between spins are of no importance on the critical dynamics [1,2]. This particularity of the dynamics is called universality. Independence of the dynamics on atomistic interactions has the dramatic consequence that the dimensionality of the exchange interactions is irrelevant for the dynamics. An even more serious consequence is that the magnetic ordering transition is not executed by the exchange interactions between spins but by the excitations of the continuous magnetic medium. The excitations of a continuous medium are delocalized bosons. RG theory could, however, not clarify the nature of the bosons. Realistic field theories of the critical dynamics therefore are not yet possible. Nevertheless a big success of RG theory was to have convincingly proven that a phase transition occurs also when interactions between spins are excluded from dynamics. This phase transition has been called stable fixed point (SFP) in order to distinguish it from the ordering temperature estimated by atomistic theories on the basis of the inter-atomic exchange interactions. The SFP generally is at a lower temperature than the predicted classical ordering temperature. In other words, magnon energies at zone boundary always are larger than conforms to the actual ordering temperature. As experiments suggest [3], the field bosons are essentially magnetic dipole radiation emitted upon precession of the magnetic moments. We have called the field bosons GSW bosons giving tribute to J. Goldstone, A. Salam and S. Weinberg [4]. Since emission and absorption of

*corresponding author; e-mail: u.koebler@fz-juelich.de magnetic dipole radiation are weak processes the approximation of freely propagating field quanta is well justified. As a consequence, field theories need to consider the energy degrees of freedom of the field exclusively. Within critical range all thermal energy is in the field. The spins receive their dynamics from the boson guiding field and only serve as indicators of the field dynamics. Since integer and half-integer spins precess differently they emit different types of field quanta. The dynamics of the field therefore is different for the two spin species. Because the actual value of the ordering temperature and the critical exponents as well are determined by the GSW boson field, the observed critical exponents should not be compared with atomistic model predictions.

\section{Analysis of experimental data}

Direct experimental observation of GSW bosons is nearly not possible. Neutron scattering fails when the bosons are mass less and, as a consequence, do not have magnetic moment. However, the finite width of the critical range convincingly proves that the dynamics of the spins is controlled by the GSW guiding field. Universality applies to the whole critical range. This is in contrast to the atomistic models for which universality applies asymptotically at the critical temperature only. It is evident that the critical exponents due to field dynamics and due to atomistic interactions must be different. Here we discuss another indirect indication of the existence of the GSW boson field. Due to the small but finite interaction between field and spins, magnon dispersions deviate surprisingly strong from predictions of spin wave theory. This interaction manifests as functional change in the magnon dispersions. Due to this functional change it can be distinguished between a boson defined low wave vector range and a magnon defined large wave vector range. Figure 1 shows as an example magnon dispersions of the $1 \mathrm{D}$ antiferromagnet $\mathrm{MnF}_{2}[5,6]$. In the wide $q$-range of $\approx 0.25<q / q_{\max }<1$ magnon dispersions 


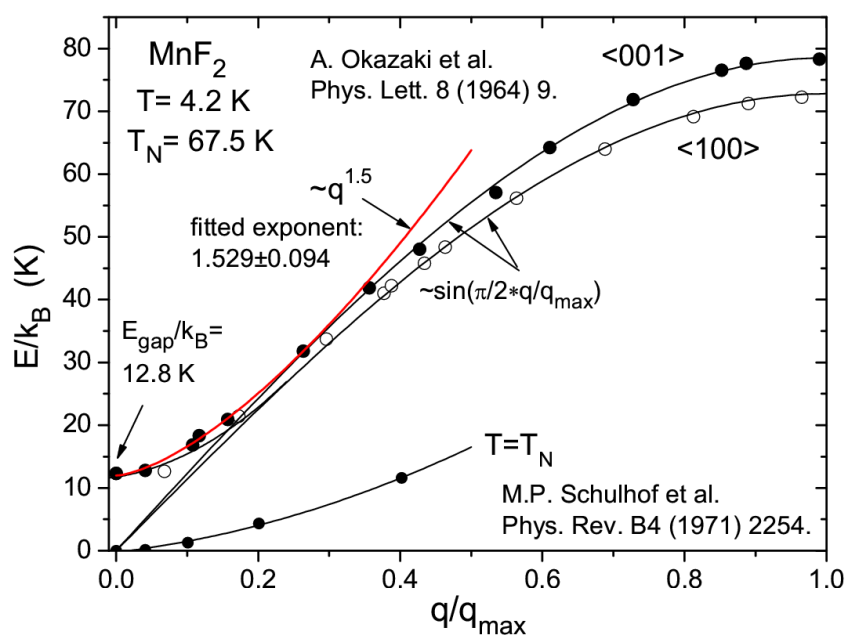

Fig. 1. Magnon dispersions of $\mathrm{MnF}_{2}$ along and transverse to tetragonal $c$-axis $[5,6]$. In the range $\approx 0.25<$ $q / q_{\max }<1$ the two dispersion branches precisely follow sine functions. At about $q / q_{\max } \approx 0.25$ abrupt deflection from sine function towards a magnon gap at $q / q_{\max } \equiv 0$ occurs. This part of the magnon spectrum is well described by $\approx q^{1.5}$ function and agrees with the dispersion of the GSW bosons. Magnons with $q / q_{\max } \neq 0$ persist into the paramagnetic phase.

precisely follow sine functions of wave vector, in formal agreement with spin wave theory. Magnon dispersions are very nearly isotropic. Isotropy of the non relevant magnons is in sharp contrast to the one-dimensional boson field (see below). For $q / q_{\max } \lesssim 0.25$ a rather sudden deflection from sine function towards a magnon gap at $q / q_{\max } \equiv 0$ occurs. For $<001>$ direction this part of the magnon spectrum is well described by gap energy plus $q^{1.5}$ function. As we could show [7], for magnets with half-integer spin and one-dimensional boson field the dispersion is $\approx q^{1.5}$. In other words magnon dispersions in the boson defined low $q$-range agree with the dispersion of the GSW bosons. This we must attribute to the interaction between magnons and GSW bosons. Condition for this interaction is that the bosons have magnetic moment (a non linear dispersion). The magnon gap also is boson defined and has identical temperature dependence as the order parameter. Due to the magnon-GSW boson interaction the extreme anisotropy of the 1D field gets transferred to the intrinsically isotropic spin system. As a consequence, the spin flop field of $\mathrm{MnF}_{2}$ is as large as $\approx 120 \mathrm{kOe}$ [8]. Magnons with $q \neq 0$ have different temperature dependence than the magnon gap and persist into the paramagnetic phase. Observation of magnons above ordering temperature conforms to the fact that thermal equivalent of magnon energy at zone boundary (the near neighbor interaction strength) generally is larger than the ordering temperature. Furthermore, magnons near zone boundary commonly exhibit weak or no anomaly at the critical temperature. This shows that they are not involved directly in the ordering process.
That the boson guiding field in $\mathrm{MnF}_{2}$ is $1 \mathrm{D}$ reveals clearly from measurements of the staggered susceptibility using elastic neutron scattering [6]. It can be seen in Fig. 2 that only the longitudinal susceptibility diverges. Typical for the boson controlled spin dynamics is that the critical power function of $\chi^{\|}$holds over a finite temperature range. The transverse susceptibility does not diverge. In other words, there is critical dynamics along tetragonal $c$-axis only. Consequently the governing boson field is $1 \mathrm{D}$. At about $T^{*}=77 \mathrm{~K} \chi^{\|}$and $\chi^{\perp}$ converge. Crossover to isotropic susceptibility marks the limit of the critical range. At this crossover thermal energy changes from field to spin system. Isotropy for $T>T^{*}$ conforms to the pure spin moment of the $\mathrm{Mn}^{2+}$ ion and proves atomistic dynamics. As a summary, in $\mathrm{MnF}_{2}$ the GSW boson field is one-dimensional but magnon dispersions are three-dimensional and isotropic. It is evident that we have to classify $\mathrm{MnF}_{2}$ as a onedimensional antiferromagnet.

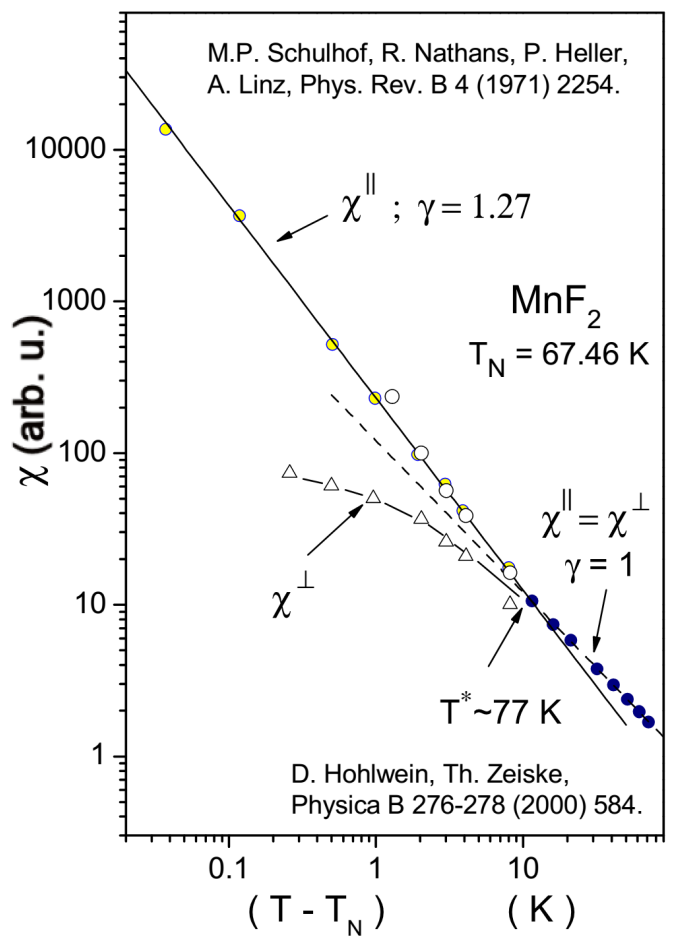

Fig. 2. Log-log plot of longitudinal and transverse staggered susceptibility of $\mathrm{MnF}_{2}$ as a function of $\left(T-T_{\mathrm{N}}\right)[6,10]$. Only the longitudinal susceptibility diverges in this $1 \mathrm{D}$ antiferromagnet. The finite width of the critical range is from $T_{\mathrm{N}}=67.46 \mathrm{~K}$ to $T^{*}=77 \mathrm{~K}$. At $T^{*}$ crossover to isotropic susceptibility occurs.

The critical power function of the order parameter also holds over a finite distance from critical temperature. This is illustrated for the rhombohedral antiferromagnet $\mathrm{Cr}_{2} \mathrm{O}_{3}$ in Fig. 3. In a plot of $[M(T) / M(T=0)]^{3}$ as a function of temperature a linear dependence results in the critical range near $T_{\mathrm{N}}$ proving critical exponent of $\beta=1 / 3$. The width of the critical range amounts to $0.2 T_{\mathrm{N}}$ and is sharply limited by the crossover 


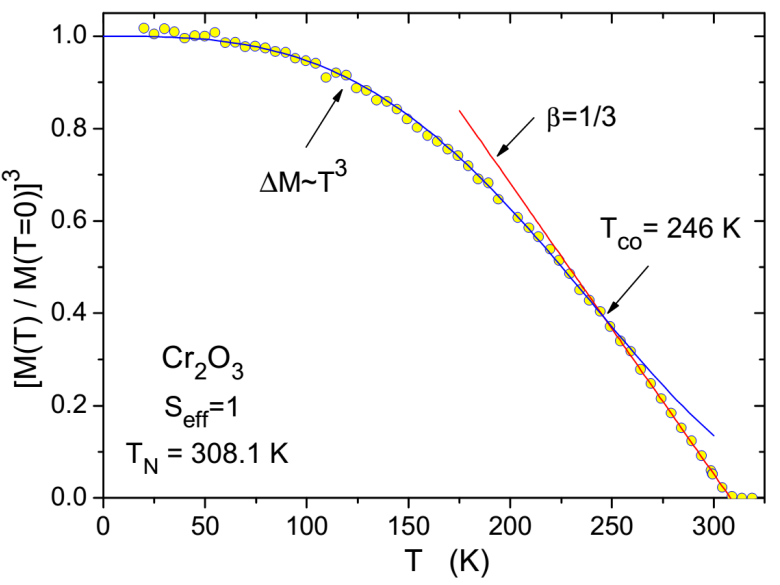

Fig. 3. Third power of reduced order parameter of $\mathrm{Cr}_{2} \mathrm{O}_{3}$ as a function of temperature. The critical range near Néel temperature spreads from crossover temperature $T_{\mathrm{CO}}=246 \mathrm{~K}$ to $T_{\mathrm{N}}=308.1 \mathrm{~K}$ Critical exponent is $\beta=1 / 3$. For $T<T_{\mathrm{CO}} M(T) / M(T=0)$ follows universal power function of type $\Delta M \approx T^{3}$. This is the universality class of $1 \mathrm{D}$ magnets with integer spin $\left(S_{\text {eff }}=1\right)$.

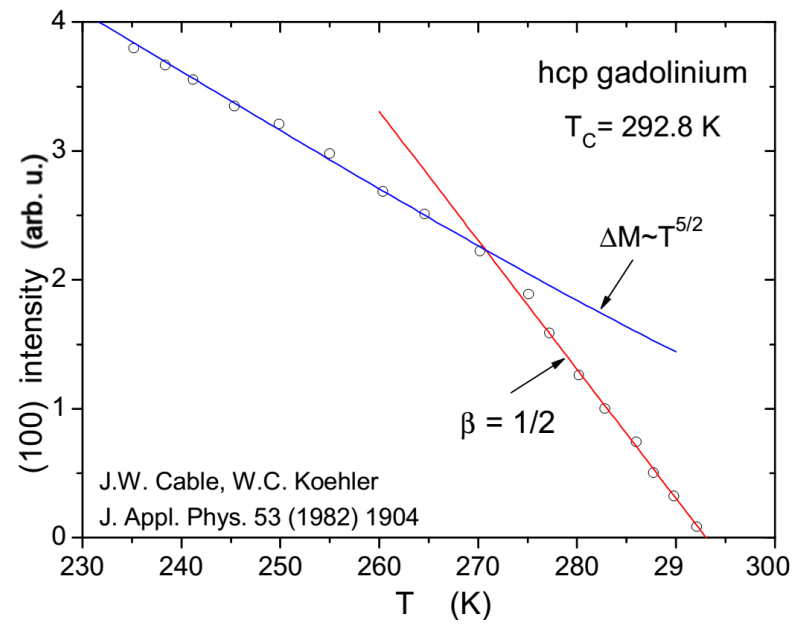

Fig. 4. Neutron scattering intensity of (100) reflection $\left(\approx M^{2}\right)$ of hcp gadolinium as a function of temperature [11]. The critical range is finite, critical exponent is $\beta=1 / 2$. Mean field critical exponent is frequently observed in isotropic magnets with half-integer $\operatorname{spin}(S=7 / 2)$.

at $T_{\mathrm{CO}}=246 \mathrm{~K}$. At $T_{\mathrm{CO}}$ the analytical change from critical power function of type $\approx\left(T_{\mathrm{N}}-T\right)^{\beta}$ to "critical" power function at $T=0$ of type $\Delta M \approx T^{\varepsilon}$ occurs. The universal exponent of $\varepsilon=3$ is typical for integer spin and one-dimensional boson field [7]. Nominally the spin of the $\mathrm{Cr}^{3+}$ ion in $\mathrm{Cr}_{2} \mathrm{O}_{3}$ should be $S=3 / 2$. Since crystal field interaction is relevant the effective spin is $S_{\text {eff }}=1$ only. $S_{\text {eff }}=1$ conforms to the observed saturation magnetic moment of $m=2.76 \mu_{\mathrm{B}}$ instead of $m \approx 3.8 \mu_{\mathrm{B}}$ commonly observed for the $\mathrm{Cr}^{3+}$ ion [9].
Ferromagnetic gadolinium with $S=7 / 2$ is a further example to demonstrate the finite critical range due to boson dynamics. Very important is that order parameter data in Fig. 4 have been obtained by zero field neutron scattering [11]. In magnetization measurements the sample is in the magnetically saturated state and, in principle, 1D. Critical exponent of $\beta=1 / 2$ is of mean field type. This does not mean that Gd conforms to conventional mean field model simply because the critical power function holds over a finite temperature range. As a conclusion, by chance it can happen that the exponents known from the atomistic models occur at SFP.

\section{Summary}

Only in Ising magnets the dynamics is atomistic and well described by classical spin models. In all ordered magnets with three-dimensional spin the dynamics is governed by a boson guiding field. The field quanta are essentially magnetic dipole radiation emitted upon precession of the magnetic moments. Consequently, magnets have to be classified by the dimensionality of the relevant boson field. Magnons are non relevant local excitations and can have a different dimensionality than the field.

\section{References}

[1] K.G. Wilson, Rev. Mod. Phys. 47, 773 (1975).

[2] E. Brézin, J.C. Le Guillou, J. Zinn-Justin J. Phys. Rev. B 10, 892 (1974).

[3] U. Köbler in: Recent Developments in Magnetism Research, Nova Science Publ. Hauppauge, N.Y. (2013).

[4] J. Goldstone, A. Salam, S. Weinberg, Phys. Rev. 127, 965 (1962).

[5] A. Okazaki, K.C. Turberfield, R.W.H. Stevenson, Phys. Lett. 8, 9 (1964).

[6] M.P. Schulhof, R. Nathans, P. Heller, A. Linz, Phys. Rev. B 4, 2254 (1971).

[7] U. Köbler, A. Hoser, Acta Phys. Pol. A 121, 1176 (2011).

[8] Y. Shapira, S. Foner, Phys. Rev. B 1, 3083 (1970).

[9] U. Köbler, A. Hoser, J.-U. Hoffmann, Physica B 382, 98 (2006).

[10] D. Hohlwein, T. Zeiske, Physica B 276-278, 584 (2000).

[11] J.W. Cable, W.C. Koehler, J. Appl. Phys. 53, 1904 (1982). 